DOI: https://doi.org/10.46630/phm.12.2020.05

Marinko T. Koščec ${ }^{1}$

Université de Zagreb

Faculté de Philosophie et Lettres

Département des études romanes
Article de recherche УДК 821.133.1.09 Chevillard É.

Reçu : le 21/2/2020

\title{
LA DÉROUTANTE LÉGÈRETÉ D’UN DÉSESPOIR LOUFOQUE : LA FICTION RÉINVENTÉE D'ÉRIC CHEVILLARD
}

Nous nous proposons d'étudier comment ce romancier s'acharne à la fois sur le réel et sur les codes littéraires, élaborant ainsi un univers singulier, invraisemblable, une sorte d'apesanteur ludique et hédoniste. Enracinée dans un monde déplaisant et dépourvu de sens, cette fiction en jubile au lieu de le déplorer ; elle abolit les référents et s'affranchit joyeusement de chronologie et de causalité, comme de la tâche mimétique. Les récits de Chevillard rejettent les contraintes de la structure narrative et évoluent essentiellement selon le mode digressif, en évitant les intrigues, en contestant la notion même d'événement, en refusant la distinction entre l'essentiel et l'insignifiant. L'écriture devient une lutte contre les conventions, contre tout ce qui est médiocre et ordinaire, au nom de l'imagination débridée. Libérée de la tâche de raconter, elle verse dans une esthétique loufoque et burlesque, mettant en dérision tout discours de maîtrise ou normalisation. L'étude démontre également que l'œuvre de Chevillard, malgré certains effets de minimalisme qui proviennent de son éthique de réduction et de déconstruction, relève d'une réinterprétation du baroque, autrement dit d'une fuite méthodique dans l'hyperbolique et le saugrenu. burlesque

Mots-clés : littérature, roman, digression, déconstruction, ludique,

\section{L'art de la fuite}

Éric Chevillard, romancier non seulement antiréaliste mais aussi traître, saboteur qui s'est infiltré dans l'espace romanesque pour mieux le détruire de l'intérieur, saborde passionnément ce qu'il appelle « romans »: il entrave, ralentit, détourne, fragmente ou pulvérise leur cours, au point d'y créer une

\footnotetext{
${ }^{1}$ mkoscec@fffzg.hr
} 
allure pathologique. ${ }^{2}$ C'est surtout le caractère débordant et délirant qui distingue son écriture, qui part dans tous les sens en prétendant avancer et ne cesse de s'inventer tout en se détruisant. En effet, la présente étude vise à démontrer que le texte de Chevillard est sous-tendu par tout un réseau de paradoxes, ambivalences ou contradictions inhérentes.

Soulignons pour commencer que très peu d'auteurs aujourd'hui jouissent d'un tel consensus critique ${ }^{3}$; cependant, dans le cas de Chevillard, l'estime ne s'accompagne nullement par la popularité. Presque sans exception, ses livres rencontrent l'enthousiasme des spécialistes mais aussi le désintérêt du grand public, en dehors d'un relativement petit nombre d'admirateurs. Ceci n'est pas si étonnant si l'on constate que les critiques saluent surtout la nature non-conformiste et subversive de cette écriture, ses gestes de rejet des chemins battus, sa mécanique loufoque fondée sur des tournures ludiques et saugrenues. ${ }^{4}$ En revanche, les aspects délirants et dysfonctionnels ne sont pas forcément ce que le fameux grand public recherche en prose.

Les journalistes littéraires de la presse généraliste ont plus que suffisamment mis en avant la virtuosité de cet auteur, mais celle-ci est une vertu à double tranchant. Emprunté au domaine musical, le terme de virtuosité est susceptible d'emprunter une signification péjorative, à savoir l'habilité technique dépourvue de talent, qui ne pourrait donc aboutir qu'à des constructions textuelles artificielles, décoratives mais creuses. Étant donné la tendance du public d'aujourd'hui de confondre art et artificialité, il n'est pas surprenant qu'on interprète la manifeste volonté artistique de Chevillard comme une pose sans substance.

Les critiques sont pourtant d'accord que cet auteur échappe à la pure virtuosité stylistique. Selon Jourde, si le texte chevillardien évite de tomber dans la catégorie d'exercice de style, c'est grâce à un facteur presque mystique, miraculeux, que l'appareil critique ne parviendra pas à saisir. ${ }^{5}$

\footnotetext{
${ }^{2}$ Dans une correspondance, l'auteur confesse qu'il ne s'est jamais considéré comme un vrai romancier, préférant s'octroyer une liberté de forme de plus en plus grande (BESSARDBANQUY et JOURDE $2015: 10-11)$.

${ }^{3}$ Selon M. Bonazzi (2013 : 150), autour de l'œuvre de Chevillard dans la presse spécialisée tant que généralisée " on ne recense aucune critique négative du style des sept romans de l'auteur publiés aux Éditions de Minuit ».

${ }^{4}$ En prenant l'exemple de Chevillard, Blanckeman affirme : «L'écrivain est celui qui usurpe une matière langagière, substituant à sa fonction d'usage des applications littérales qui regorgent de tours systématiques et de passe délurées dont provient une certaine essence du romanesque » (2014: 12).

${ }^{5}$ « Les livres sur rien, comme ceux de Chevillard, qui tiennent à la seule force du style, sont toujours menacés par la redite, l'auto-caricature, la gratuité ou le maniérisme. Il semble qu'audelà d'une certaine limite dans le travail des mots, il ne reste plus que le fragment (tentation permanente chez Chevillard) ou le silence. Pourtant, à chaque fois, le miracle a lieu » (Jourde $2002 b: 8-9)$.
} 
Cependant, Blanckeman maintient que le texte de Chevillard échappe à la seule virtuosité stylistique «[...] parce qu'il s'approprie la tradition moderniste à des fins propres. L'écrivain s'attache à subvertir les règles et les usages de la logique issue des traditions aristotélicienne et cartésienne en les accomplissant à l'extrême » (2002 : 79). Dans le même temps, ceci n'est que l'un de nombreux aspects de l'écriture de Chevillard, décrits ci-dessous, qui permettraient d'illustrer le postmodernisme littéraire, si cet écrivain ne rejetait pas vigoureusement l'appartenance à tout -isme.

Souvent, c'est par paresse ou parce qu'il ne sait pas à quoi attribuer les qualités intrinsèques qu'il devine, que l'observateur les ravale au style, notion d'autant plus problématique qu'elle revêt volontiers l'acception d'une couche superficielle, plus ou moins décorative, qui servirait à substituer des considérations plus profondes. Le concept des exercices de style, qui renvoie d'un côté aux gammes pratiquées en milieu scolaire et de l'autre à l'Oulipo, semble particulièrement déplacé à propos de notre auteur car celuici revendique le naturel, la spontanéité, voire la naïveté, autrement dit l'anticontrainte et l'anti-formalisme. Voilà comment il rejette l'affinité avec les principes d'écriture oulipiens, les règles et protocoles : « Si je récuse la notion d'exercice, c'est qu'elle sous-entend une conscience extrêmement aiguë de ce qu'on fait, la mise en œuvre d'un procédé conçu préalablement, alors que j'écris avec une certaine innocence ce que je dois écrire. La phrase s'emballe, elle accueille tout ce qui va lui permettre de se développer. Elle veut durer, elle progresse en sinuant, comme un animal toujours en quête de nourriture » (BONAZZI 2013b : 195).

Par cela même, l'auteur confirme la nature centrifuge, digressive de sa phrase, qui ne cesse de s'éloigner de ce qu'elle propose comme son sujet. En effet, il présente ce qui est mal à propos, illogique et insolite, comme « la nourriture » favorite de son énoncé, qui semble propulsé surtout par le désir de surprendre, de déconstruire toute attente ou dessein prétabli. Le sens est constamment en fuite ; le discours adopte volontiers les contours d'une argumentation logique et sévère, mais par des glissements infimes et d'incessantes métamorphoses, il finit par soutenir n'importe quoi ou tout ce qu'on voudra, donc en réalité rien. À titre d'exemple, un des romans tourne autour d'un animal énigmatique nommé Palafox, qui se voit attribuer des caractéristiques différentes, dont certaines s'excluent mutuellement, ce qui l'empêche de devenir un seul animal et de se montrer dans le texte. En singeant des énoncés disparates (judiciaire, administratif, policier, publicitaire, journalistique, etc.), ce roman devient un amalgame loufoque. Dans l'ensemble de l'œuvre, les registres, les perspectives et les facettes de sens se multiplient parfois au point qu'il est impossible d'identifier la position auctoriale. ${ }^{6}$

${ }^{6}$ Selon Blanckeman, « Des histoires se juxtaposent, s'entrecroisent et se superposent ainsi en 
Dans le texte chevillardien, la fuite s'opère principalement par la digression, instrument privilégié de subversion et espace de jubilation créatrice. Le discours est soumis à ce que l'auteur, dans Du hérisson, appelle " une dynamique dilatoire ». Bien qu'elle puisse être perçue, du point de vue de l'économie narrative, comme une maladie de la linéarité, elle obtient un rôle fondamental : «Je ne serais point surpris [...] que cela même qui entrave mes gestes et ralentit mon travail soit une pièce maîtresse du système en vigueur grâce à quoi tout le reste roule » (2002 : 82). Dans « tout le reste », évidemment, il faut compter l'histoire, l'intrigue, l'action, etc. Tant mieux si elles se produisent, mais ce seront plutôt des accidents de la route. On dirait que la narration de Chevillard, lorsqu'elle se déroule paisiblement, n'est qu'en attente de la prochaine vague digressive. En fait, ses détours sarcastiques déconstruisent même la notion de digression (JULY 2013 : 73). Évidemment, l'extase dilatoire accompagnée de la propension métanarrative risque de virer à la logorrhée, c'est-à-dire au bavardage, mais selon notre auteur les vrais livres sont justement ceux « [...] où on n'avance pas, desquels on ne sort pas, on ne voit pas le bout et on prolonge indéfiniment la lecture en l'interrompant souvent » (CHEVILLARD 1994 : 234). Le raffinement des techniques dilatoires manifestement procure du plaisir à l'écrivain ; la tâche difficile, c'est de partager cette passion avec le lecteur. ${ }^{7}$

Dans la pratique textuelle, la digression vire facilement à la régression. Plusieurs narrateurs prétendent avancer, voire vivre à reculons : « [...] revenons plutôt sur nos pas [...] rebroussons chemin - la nuque, le dos, les fesses et les talons n'y sont pas opposés, ils ralentissent autant qu'ils peuvent la fuite en avant suicidaire du corps irrésistiblement attiré par le vide » (CHEVILLARD 2001 : 124). Ceci n'est que l'une de très nombreuses représentations de la volonté d'écrire à contre-courant, en défiant les atteintes et les logiques canoniques. De même, on verra les personnages marcher sur les mains, vivre accrochés au plafond d'un appartement bourgeois ou avec une chaise renversée sur la tête. Le renversement des choses peut dévoiler leur vacuité ou déviations cachées ; l'irruption des éléments insolites au milieu

une seule et même structure d'égarement qui multiplie des niveaux de situations romanesques différents, des jeux de réverbération critique et des lignes de fuite autobiographiques ouvrant autant de perspectives illusoires vers une identification possible de l'auteur » (2014:25). Viart confirme : «Pris dans les rets d'une saturation autoréflexive, le lecteur ne peut que s'abandonner passivement aux fantaisies, digressions et escapades du texte » (2014:72).

${ }^{7}$ On pourrait qualifier cette propension d'exhibitionniste : «Il y a en vérité comme un plaisir exhibitionniste dans cet éclat de la digression, dans l'évidence de sa finalité amplificatrice, comme si ce n'était au fond dans ce recoin du texte que la pureté originelle de l'écriture, le désir d'écrire sans objet, sans autre projet que de dire le monde dans sa plus éclatante incongruité, pouvait le mieux se laisser apercevoir, sans pudeur, sans hypocrisie » (BESSARD-BANQUY 2003 : 42), mais elle peut être perçue aussi comme sa qualité principale : « les broderies de Chevillard sont donc bien l'essentiel du tissu : on ne lit que pour sa dentelle » (JULY 2013 : 75). 
du texte est une diversion qui fait deviner le hasard dans ce qu'on fait passer pour l'ordre et la nécessité. ${ }^{8}$ Chez Chevillard, les incongruités ne sont pas des ingrédients accidentels parsemés afin de pimenter la texture ; comme on verra par la suite, elles participent à la stratégie globale d'attaque - contre le réel en tant que prison de la conscience et contre la littérature en tant que sa simple reproduction.

\section{L'art de la contre-attaque}

« Mes livres sont tous habités par une espèce de colère ou de hargne. Toute tentative littéraire ou poétique vise à réformer l'ordre des choses, à ordonner un monde où la parole performative, toute-puissante, substituerait ses injonctions et ses rythmes aux structures du système en vigueur ", affirme l'auteur (ALLEMAND 2010). Malgré l'innocence dont il se réclame ailleurs, le texte chevillardien recèle un projet intellectuel, qui n'est rien de moins que la réinvention du monde, fût-elle parodique. Bien que le comique chevillardien frôle volontiers l'infantilisme et le non-sens, on y reconnaît la posture de résistance : l'ensemble de cette entreprise fictionnelle est fondé sur le principe de rejet moqueur ou méprisant de tout ce qui le mérite. La liste en est évidemment longue, depuis les inconvénients comme le vieillissement et la mort, en passant par ce qui dérange l'artiste dans ses efforts quotidiens, et jusqu'aux différents aspects de la modernité sociale, technique ou scientifique aussi bien que littéraire.

Clairement, nous ne vivons pas dans le meilleur des mondes possibles, mais cet écrivain ne se contente pas de hausser les épaules ni de lamenter. Il passe à la contre-attaque, qu'il cultive comme un véritable art. Ne comptant pas ses pertes ni les victimes collatérales, il mène une guerre incessante contre ce qui est ordinaire, banal ou médiocre au monde, surtout lorsque cela se fait passer pour une valeur, en particulier dans le domaine littéraire. Sa prose découle largement d'un désir de vengeance; elle pourchasse et pourfend les apparences du narcissisme, de l'imposture, du cliché creux, de l'insensibilité, de la bêtise, etc. Les cibles fluctuent, évoluent, certaines retournent et s'érigent en obsessions, telle la figure du critique littéraire mesquin, ou de l'écrivain raté (que "le système " glorifie parfois comme héros national, mais aussi qu'on risque soi-même de devenir). Dans le même temps, l'auteur

\footnotetext{
${ }^{8}$ On pourrait y lire un certain anarchisme, une volonté de déconstruction gratuite, ou bien une jouissance régressive de plongée dans le chaos originel, «comme si le plaisir le plus intense et le plus libre ne pouvait se donner libre cours qu'en défaisant ce qui est construit, en arrêtant ce qui évolue » (JOURDE 1999 : 302). Or, Blanckeman souligne : « Il s'agit moins en cela d'enrayer le système que de le laisser aller à sa fantaisie sur d'autres rails pour explorer en hurluberlu des ordres du réel insoupçonnés » (2014:30-31).
} 
invoque la complicité du lecteur par d'étranges amalgames, comme celui de la (feinte) mégalomanie et de l'autocastration. Dans La Nébuleuse de $\mathrm{Crab}$, le protagoniste écrit son Grand-Euvre en combinant tous les mots du dictionnaire, en créant ainsi un livre gigantesque, pour ne garder finalement que les cent meilleures pages.

Au niveau du matériau langagier, Chevillard s'attaque à l'arbitraire du signe et à l'usure des mots, en démontant les locutions figées, en ranimant les métaphores mortes et en détournant les étymologies. Il est évident que la simple narration des histoires et le paisible discours mimétique ne l'intéressent pas. ${ }^{9}$ En revanche, il aime bien se moquer de " l'effet de réel », c'est-à-dire démontrer l'arbitraire de la construction romanesque. Son écriture se coupe volontiers de toute entrave réelle et rationnelle, afin de conduire le texte vers un point de dépassement ontologique, un point-limite où il cesserait d'être lui-même afin de « devenir sa propre transcendance, une sorte de conscience suraiguë de lui-même » (BESSARD-BANQUY 2003 : 138). En effet, comme le souligne Jourde, le texte y parvient facilement : " Dans la jonglerie de Chevillard, tout se passe comme si les choses les plus ordinaires devenaient capables, grâce à un peu d'habileté (et dans la mesure où cette habileté se moque d'elle-même), de se transcender » (1999b : 273).

Voilà comment l'auteur présente une de ses stratégies narratives en parlant d'un autre alter-égo écrivain : "Pour conduire et endiabler ses récits, il compte sur les accélérations délirantes que favorise son goût du discours logique poussé jusqu'à ses plus extrêmes conséquences et conclusions, bien au-delà de celles auxquelles, avec sagesse, avec prudence, avec sa sagesse ennuyeuse et sa mesquine prudence, s'arrête la raison » (CHEVILLARD 2012 : 78). Au cœur de cette entreprise fictionnelle, il y a donc un procès intenté contre le Logos, dont l'enjeu serait de donner une version littéraire de l'audelà de la raison en désaxant les récits et les discours, en remplaçant ce que nous sommes habitués à appeler le réel par des réalités parallèles.

Il s'agit d'explorer le pouvoir des mots à la fois contre l'ordre des choses et contre le vide. Afin de les déconstruire, l'auteur emprunte différentes recettes narratives : récit de voyage, roman d'aventures, conte, mythologie ou légende, édition critique, (fausse) biographie, (fausse) autobiographie, journal, portrait moraliste, autofiction... Il prend toutes ces formes pour références, mais s'en affranchit en les poussant au-delà de leurs limites, et les gonflant jusqu'à la caricature et en substituant l'objet par l'autoréflexion. Prenons l'exemple d'Oreille rouge : le modèle romanesque et le modèle du récit de voyage, que le texte prend pour références, devraient en principe suffire à assurer un

${ }^{9}$ Bessard-Banquy remarque : « Ses histoires d'ailleurs commencent rarement bien et finissent souvent mal, comme si le romanesque ne pouvait mener qu'à la catastrophe » (2003:138). On pourrait ajouter que certaines commencent plusieurs fois mais jamais vraiment, et qu'au lieu de se terminer proprement, elles inclinent à s'interrompre à un moment aléatoire. 
minimum de narrativité. Cependant, le récit ne se matérialise que sur le mode de la dérision et du refus ; il ne fait que déconstruire les modèles narratifs qu'il prétend suivre. À tout début, le narrateur récuse la légitimité de son énoncé en contestant la possibilité que quelque chose se passe qui puisse fournir prétexte et matière à récit. Le postulat fondamental, c'est que dans le monde d'aujourd'hui il ne peut plus y avoir de voyage, et par conséquent, encore moins de récit qui le relate. Le motif paradigmatique est celui de la poursuite des hippopotames au Mali, donc une version rabaissée de la quête romantique. Du début à la fin, le héros proteste contre l'idée même d'un déplacement, mais voir les hippopotames donnerait peut-être une tout petite légitimation à ce voyage, ne serait-ce qu'une miette de sens. Évidemment, l'hippopotame s'avère introuvable; le protagoniste repartira bredouille car l'animal est une incarnation métaphorique du récit impossible. Par glissement métonymique, sa disparition s'étend sur tout le réel, qui devient un horizon impossible à rejoindre car ce récit de voyage échoue entièrement à en rendre compte. Par cela même, le lecteur est invité à reporter son attention sur la seule réalité produite par le travail d'écrivain, c'est-à-dire le texte.

Il ne serait peut-être pas faux d'affirmer que Chevillard écrit au lieu du réel et contre le réel, pour l'évacuer et pour s'en venger, de son impact nocif sur l'imagination créatrice et sur la lucidité.

\section{Drôle de minimalisme ou minimalisme drôle}

Aussi frivole et gratuit qu'il puisse paraître, l'humour est chez Chevillard toujours doublé de mélancolie ou d'angoisse. Sa prose à la fois désinvolte et solennelle hésite et oscille constamment entre la taquinerie et la complainte, la farce et le désespoir ; il en découle un rire grinçant ou une morosité hilarante, ce qui a valu à l'auteur le titre du « plus rigolard de nos poètes désespérés ou le plus triste de nos romanciers comiques » (BESSARDBANQUY 2003 : 20). L'essentiel y est inséparable du trivial, le penchant encyclopédique des fabulations poétiques. L'éloquence grave débouche sur la bouffonnerie ou le sarcasme, le questionnement ontologique est toujours ponctué par des paradoxes irrésolubles, l'ironie désillusionnée alterne avec l'emphase de l'espoir.

De même, tout en dénonçant sans cesse la littérature comme encombrante et intenable, les narrateurs chevillardiens perpétuent sa défense. Ils évoquent l'écriture comme une activité sublime, suprême, voire seule capable de bâtir l'être. La langue est prise pour un chantier permanent, un terrain de construction et d'expérimentation sans règles ni bornes. Par cela même, elle devient un immense champ de plaisir, car cette exploration est souvent ludique 
et humoristique, fondée sur la conviction qu'il ne pourrait pas y avoir de salut sans rire. Ce comique affranchi des contraintes du sens et de la fonctionnalité classique produit une littérature délivrée de l'obligation de refléter le monde. ${ }^{10}$

D'un autre point de vue, l'humour apporte un antidote au sérieux et à la souffrance qui infectent la vie ; l'esprit ludique permet de dédramatiser la lutte existentielle. Les narrations chevillardiennes sont construites autour soit des drames ridiculisés, soit des traumatismes drolatiques. Déjà le premier roman, Mourir m'enrhume, badine avec les derniers jours d'un vieillard et se moque de la mort. À l'inverse, dans le plus récent, L'Explosion de la tortue, la mort d'un petit animal domestique déclenche une mise en question générale et ébranle les fondements de l'existence du narrateur.

Sauf quelques exceptions, les protagonistes sont chez Chevillard à peine esquissés, des hommes sans rien d'extraordinaire, souvent insignifiants. Les événements sont rares, si l'on ne compte que les mouvements physiques capables de renverser la situation narrative. En revanche, fleurissent les événements discursifs, jeux de mots et autres figures rhétoriques, comme si le texte était porté par le désir d'écrire sans objet, dans le seul but de le déployer et d'admirer sa croissance.

Or, cela ne l'empêche pas de s'apparenter au minimalisme, dans la mesure où celui-ci rejette le modèle balzacien du réalisme psychologique, la « téléologie événementielle » ou la « vectorisation du texte vers son dénouement » (AUDET 2007). Le terme de minimalisme est applicable sur Chevillard également au sens de la focalisation du détail insignifiant, et lorsqu'il implique « la mise en scène insistante des réticences du narrateur par rapport à sa propre activité diégétique » (THIBAULT $2012: 121$ ). En revanche, on y est aussi loin du « récit minimal » au sens de la réduction (PRINCE 2012:24), que de « l'écriture du quotidien » et son éloge du presque rien (BERTRAND 2005 : 16), ou de la " littérature blanche » et son désinvestissement, sa recherche du degré zéro de la rhétorique (JOURDE 2002, RABATÉ et VIART 2009). Par rapport à ce dernier aspect, Chevillard est en fait plus proche du pôle baroque. Ses versions du « livre sur rien» sont exubérantes, extravagantes et enjouées. Son récit est retourné ; il exhibe sans gêne sa doublure métatextuelle au lieu de tisser des histoires. Il dévoile non seulement l'architecture du texte, mais aussi les soubassements de la conscience qui s'interroge sur ellemême ${ }^{11}$. Cette écriture est donc fort spéculative, autrement dit auto-érotique, puisqu'elle aime bien se contempler dans le miroir de son épanouissement débridé.

\footnotetext{
${ }^{10}$ Or, pour cela même, elle est souvent reléguée dans les marges, parce que le ludique, le comique et l'incongru sont perçus comme indignes des œuvres majeures. Jourde affirme : « Cela ne peut que s'aggraver lorsque ce type de comique ne paraît pas avoir de fonction bien précise. Passe encore pour la parodie ou la satire, qui ont leur objectif et même leur utilité ». Par contre, « des hurluberlus (...) auront toujours du mal à se faire reconnaître comme de véritables écrivains, dès lors qu'ils ne peuvent présenter une caution sérieuse qui s'appellerait surréalisme ou tout autre nom en -isme » (1999: 300). ${ }^{11}$ Selon Viart, « son imaginaire est le fruit de ce retournement exploratoire du langage sur luimême » (2014: 79).
} 
Cela dit, soulignons que l'amour-propre y est inséparable de l'autodérision et de l'autodestruction. Le sabotage participe à la constitution de l'espace textuel. La décomposition est l'état normal des choses ; la phrase déconstruit volontiers sa propre logique. ${ }^{12}$ C'est une prose qui parodie ses moyens stylistiques et rhétoriques favoris en les abusant au-delà du maniérisme. Écrire, entre autres, y sert à lutter contre soi-même, contre les enflures de l'ego et toute complaisance. Les narrateurs dénoncent souvent la vanité égoïste des littérateurs. Comme déjà observé plus haut, nombreuses sont des figures caricaturales de l'écrivain, pédant, présomptueux, jaloux, haineux, vindicatif et surtout frustré, car « incapable d'élaborer un personnage ex nihilo, incapable d'exister en dehors de ses personnages et entièrement consumé par cette entreprise, somme toute de discrédit de la fiction » (NARJOUX 2013 : 54). Certaines de ces représentations de l'échec auctorial aboutissent à l'impossibilité d'écrire, comme dans $D u$ hérisson, où le sujet autobiographique ne parvient même pas à constituer un « moi » cohérent.

La cohérence ne figure pas parmi les priorités dans le texte chevillardien, que déjantent sans relâche des effets de loufoque. Toute incongruité y est bienvenue pour dévoiler des aspects insoupçonnés et déroutants du monde. ${ }^{13}$ Dans cet objectif, l'écriture se livre à la rhétorique parodique de l'amplification ou de l'invective fielleuse, à l'excès de précision et au gonflement des détails (souvent insignifiants), à l'exagération syntaxique à travers la multiplication de subordonnées relatives, à la suppression des données nécessaires, à l'interprétation au pied de la lettre, à la distorsion de l'ordre sémantique et causal, au délire logique, au pur non-sens. Parmi les figures incongrues les plus représentées, on trouvera des comparaisons, métaphores et métonymies excessives ou/et grotesques, des synecdoques impertinentes, des parabases et autres glissements métaleptiques, des fatrasies et fatras, des énumérations où intervient le procédé de coq-à-l'âne et de chimère accidentelle ou essentielle, etc. ${ }^{14}$ Tout cela, malgré une apparence chaotique, sont des instruments méthodiquement utilisés pour engendrer des variations

\footnotetext{
${ }^{12}$ André le résume par la formulation suivante : «Une écriture du moindre qui s'emploie à opérer une déconstruction systématique des éléments de narrativité et une réduction non moins systématique de son contenu narratif, inventant par là un récit minimal à la mode chevillardienne qui, placé sous le signe de ce qui peut se définir paradoxalement comme une énergétique de la pulvérisation, s'invente en se détruisant et se défait en avançant » (2012:137).

${ }^{13}$ Voilà comment Jourde explique la diversion cachée dans l'irruption de l'incongruité : « Le texte incongru parle en termes très concrets et précis de rien, gonfle une bulle de vide [...] Et finalement, on est souvent amené à se demander de quel côté est le vide : on pensait d'abord que ce petit objet stupidement précis n'avait rien à faire là, on finit par soupçonner tout le reste d'être vain et idiot par rapport à lui » (1999: 303).

${ }^{14}$ Pour une classification détaillée des procédés incongrus, voir Jourde (idem).
} 
du « texte de jouissance $»^{15}$, en vue de répandre l'indétermination, semer le trouble, miner l'ordre endormant de la littérature mimétique et consensuelle.

Proclamé incernable, inclassable, indécidable par la critique, l'œuvre d'Éric Chevillard fuit en effet les étiquettes, les courants établis et la théorie. Ce texte se dérobe à l'interprétation car il est en perpétuelle mutation, instable au point de parfois paraître dénué de sujet, une pure dérive digressive. Guidé par le désir d'échapper au prévisible et au défini, il refuse de raconter les histoires qu'il promet et subvertit les recettes narratives ainsi que les modes de raisonnement canoniques. Il remplace la matière habituelle des choses par des contenus invraisemblables, favorise l'insolite, introduit des situations inadmissibles. Il explore les alternatives au réel, les champs du possible, le potentiel inédit des signes. Voici une de nombreuses explicitations de cette intention : « Mes romans voudraient instaurer un temps hors de l'Histoire, propice au songe, à une méditation poétique sans enjeu concret » (BENHAÏM 2004 : 185). Il s'agit d'un pari littéraire extrêmement risqué ; en rejetant les attaches à son époque et au contexte social, en s'affranchissant des contraintes de la fonctionnalité et du sens, on aboutit à une littérature aérienne et parfois légère comme de l'air. Dotée de sa propre matérialité, elle flotte au-dessus du « réel », dans une sphère autonome, mais qui par cela même passe souvent au-dessus des attentes des lecteurs, restant incomprise, invisible. Cependant, elle est propulsée par une joie contagieuse pour celui qui sait la reconnaître : joie de la vengeance contre la médiocrité, de la déconstruction des valeurs imposées et de toute position fixe. Bien qu'elle mine ses propres fondements par l'autodérision, l'écriture chevillardienne érige un immense édifice virtuel, incongru et absurde, un monument ironique à l'imagination intelligente, critique, ludique et jouissive.

\section{Bibliographie}

Sources

CHEVILLARD 1987 : CHEVILLARD, Éric. Mourir m'enrhume. Paris : Éditions de Minuit, 1987.

CHEVILlard 1990 : CHEVILlard, Éric. Palafox. Paris : Éditions de Minuit, 1990.

CHEVILLARD 1993 : CHEVILLARD, Éric. La nébuleuse du crabe. Paris : Éditions de Minuit, 1993.

CHEVILLARD 1994 : CHEVILLARD, Éric. Préhistoire. Paris : Éditions de Minuit, 1994.

\footnotetext{
${ }^{15}$ « Le texte de jouissance », que Barthes oppose au « texte de plaisir », serait « celui qui met en état de perte, celui qui déconforte (peut-être jusqu'à un certain ennui)» (1973).
} 
CHEVILLARD 1997 : CHEVILLARD, Éric. Au plafond. Paris : Éditions de Minuit, 1997.

CHEVILLARD 2001 : CHEVILLARD, Éric. Les absences du capitaine Cook. Paris : Éditions de Minuit, 2001.

CHEVILLARD 2002 : CHEVILLARD, Éric. Du hérisson. Paris : Éditions de Minuit, 2002.

CHEVILLARD 2005 : CHEVILLARD, Éric. Oreille rouge. Paris : Éditions de Minuit, 2005.

CHEVILLARD 2012 : CHEVILLARD, Éric. L'auteur et moi. Paris : Éditions de Minuit, 2012.

CHEVILLARD 2019 : CHEVILLARD, Éric. L'Explosion de la tortue. Paris : Éditions de Minuit, 2019.

Ouvrages théoriques

ALLEMAND 2010 : ALLEMAND, Roger-Michel. «Éric Chevillard : Choir " sans intention » - mais vers le haut ». <www.revue-analyses.org >, vol. 5, $\mathrm{n}^{\mathrm{o}}$ 1, hiver 2010. Ottawa.scholarportal.info. 16. 01. 2020.

ANDRÉ 2012 : ANDRÉ, Marie-Odile. « Pour un voyage minimal ? Oreille rouge d'Éric Chevillard ». Le récit minimal. Du minime au minimalisme. Bedrane, Sabrinelle, Revaz, Françoise, Viegnes, Michel (dir.). Paris : Presses Sorbonne nouvelle, 2012.

AUDET 2007 : AUDET, René. « Fuir le récit pour raconter le quotidien : modulations narratives en prose contemporaine ». Temps Zéro. Écritures contemporaines. Poétiques, esthétiques, imaginaires. Tempszero. contemporain.info. 17. 9. 2019.

BARTHES 1973 : Le plaisir du texte. Paris : Éditions de Minuit, 1973.

BENHAÏM 2004 : BENHAÏM, André. « Questions de préhistoire : Entretien avec Éric Chevillard ». Benhaïm, André et Lantelme, Michel (dir.). Écrivains de la préhistoire. Toulouse : P. U. Mirail, 2004.

BERTRAND 2005 : BERTRAND, Rémi. Philippe Delerm et le minimalisme positif. Paris : Éditions du Rocher, 2005.

BESSARD-BANQUY 2003 : BESSARD-BANQUY, Olivier. Le roman ludique. Paris : Presses Universitaires du Septentrion, 2003.

BESSARD-BANQUY et JOURDE 2015 : BESSARD-BANQUY, Olivier et Jourde, Pierre (dir.). Éric Chevillard dans tous ses états. Paris : Classiques Garnier, 2015.

BLANCKEMAN 2002 : BLANCKEMAN, Bruno. Les Fictions singulières, étude sur le roman français contemporain. Paris : Prétexte éditeur, 2002.

BLANCKEMAN 2014 : BLANCKEMAN, Bruno. «L'Herméneutique du fou ». Pour Éric Chevillard. Paris : Minuit, 2014. 
BONAZZI 2013 : BONAZZI, Mathilde. «Les représentations métaphoriques dans la critique littéraire de la pratique stylistique d'Éric Chevillard : comment suggérer que le style s'auto-caricature jusqu'à la provocation ? ». Narjoux, Cécile et Jollin-Bertocchi, Sophie (dir.). La langue de Chevillard ou "le grand déménagement du monde ». Dijon : Éditions Universitaires de Dijon, 2013.

BONAZZI 2013b : BONAZZI, Mathilde. «Une espèce de transe stylistique ». La langue de Chevillard ou "le grand déménagement du monde ". Narjoux, Cécile et Jollin-Bertocchi, Sophie (dir.). Dijon : Éditions Universitaires de Dijon, 2013.

JOURDE 1999 : JOURDE, Pierre. Empailler le toréador. L'incongru dans la littérature de Charles Nodier à Éric Chevillard. Paris : José Corti, 1999.

JOURDE 1999b : JOURDE, Pierre. « L'œuvre anthume d'Éric Chevillard ». Critique $\mathrm{n}^{\circ} 622$ (mars 1999) : 273-310.

JOURDE 2002 : JOURDE, Pierre. La littérature sans estomac. Paris : L'esprit des péninsules, 2002.

JOURDE 2002b : JOURDE, Pierre. « Le hérisson universel ». La Quinzaine littéraire $\mathrm{n}^{\circ} 828(01.04 .2002): 8-17$

JULY 2013 : JULY, Joël. « Chevillard : Jeu digresse donc je progresse ». La langue de Chevillard ou "le grand déménagement du monde ». Narjoux, Cécile et Jollin-Bertocchi, Sophie (dir.). Dijon : Éditions Universitaires de Dijon, 2013.

NARJOUX 2013 : NARJOUX, Cécile. « 'Instaurer un temps hors de l'Histoire' : Éric Chevillard et ses mondes possibles ». La langue de Chevillard ou «le grand déménagement du monde ». Narjoux, Cécile et Jollin-Bertocchi, Sophie (dir.). Dijon : Éditions Universitaires de Dijon, 2013.

PRINCE 2012 : PRINCE, Gerald. " Récit minimal et la narrativité ». Le récit minimal. Du minime au minimalisme. Paris : Presses Sorbonne nouvelle, 2012.

RABATÉ et VIART 2009 : RABATÉ, Dominique et Viart, Dominique (dir.). Ecritures blanches. Saint-Étienne : Publications de l'Université de SaintEtienne, 2009.

REGGIANI 2008 : REGGIANI, Christelle. " Poétiques de l'adresse, hermétisme ou idiotie ». Éloquence du roman. Rhétorique, littérature et politique aux XIXe et XXe siècles. Genève : Droz, 2008.

THIBAULT 2012 : THIBAULT, Bruno. « Les bâtons rompus de l'écriture : "'l'histoire brisée' entre récit minimal et récit minimaliste ». Le récit minimal. Du minime au minimalisme. Bedrane, Sabrinelle, Revaz, Françoise, Viegnes, Michel (dir.). Paris : Presses Sorbonne nouvelle, 2012.

VIART 2014 : VIART , Dominique. « Littérature spéculative ». Pour Éric Chevillard. Blanckeman, Bruno, Samoyault, Thiphaine, Viart, Dominique, Bayart, Pierre. Pour Éric Chevillard. Paris : Éditions de Minuit, 2014. 
Marinko T. Koščec

\section{ZAČUDNA LAKOĆA ŠA ŠAVOG BEZNAĐA: NEKA DRUKČIJA FIKCIJA ERIKA ŠEVIJARA}

Studija istražuje kako se ovaj romanopisac obračunava s realnošću i s književnim kodovima, stvarajući jedinstven, začudan prostor koji svojim ludizmom i hedonizmom prkosi gravitaciji. Ukorijenjena u neugodan i besmislen svijet, njegova fikcija pronalazi radost umjesto da oplakuje ono što nedostaje; oslobađa se referencija, kronologije i kauzalnosti, kao i mimetičkih zadataka. Odbacuje ograničenja pripovjedne strukture, oslanja se pretežno na digresije, odbija razlikovati esencijalno od beznačajnog te osporava čak i sâm koncept događaja. Pisanje postaje borba protiv konvencija, protiv svega običnog i nedovoljnog, u ime razuzdane imaginacije. Oslobođeno dužnosti da pripovijeda, ono otklizava u šašavu i burlesknu estetiku, ismijavajući svaki autoritativan ili normativan diskurs. Studija također pokazuje da Ševijarovo djelo, usprkos određenim minimalističkim aspektima koji proistječu iz etike redukcije i dekonstrukcije, predstavlja reinterpretaciju baroka, drugim riječima metodički bijeg u hiperbolu i apsurd.

Ključne riječi : književnost, roman, digresija, dekonstrukcija, burleska 